\title{
Electromagnetic Emission-aware Scheduling for the Uplink of Multicell OFDM Wireless Systems
}

\author{
Yusuf A. Sambo, Member, IEEE, Fabien Héliot, Member, IEEE, and Muhammad Ali Imran, Senior Member, IEEE
}

\begin{abstract}
The increasing demand for data and multimedia services, as well as the ubiquitous nature of the current generation of mobile devices have resulted in continuous network upgrades to support an ever-increasing number of users. Given that wireless communication systems rely on radiofrequency waves, the electromagnetic (EM) emissions from these systems are increasingly becoming a concern, especially in terms of adverse health effects. In order to address these concerns, we propose a novel resource allocation scheme for minimizing the EM emission of users in the uplink of multicell OFDM systems, while ensuring quality of service. Our scheme is based on the assumption that long-term channel state information of all the users in the network is available. A new multicell user grouping that uses the received interference powers of the users of different sectors is proposed. Furthermore, we propose two power allocation algorithms to minimize EM emission. The first power allocation algorithm performs multicell iterative optimization to obtain the transmit powers of each user in the system. On the other hand, our second power allocation algorithm uses the average channel gains of the users of different sectors to obtain an approximation of the transmit power of each user without multicell iterative optimization. As a result, this approach has a reduced complexity when compared to our first power allocation algorithm. Simulation results show that our scheme reduces EM emission by up to $70 \%$ when compared to a single cell EM emission aware scheme and by over 3 to 4 orders of magnitude when compared to spectral efficiency maximization schemes.
\end{abstract}

Index Terms-Electromagnetic (EM) emission, multicell, OFDM, power allocation, subcarrier allocation.

\section{INTRODUCTION}

There has been a tremendous evolution in mobile communications over the past few decades driven by data rate improvement at first, and more recently by energy efficiency (EE) which is gradually taking center stage in the research on mobile communications. Evolution is more than ever on the agenda, with the ongoing development of the 5th Generation $(5 \mathrm{G})$ of mobile communication systems. $5 \mathrm{G}$ will allow more devices to be connected and will rely on 10 or 100 thousands more access points to do so, and as such, the electromagnetic (EM) emissions and the serious concerns attached to them would also increase. Although there are no established cases of short term health effects of exposure to EM emissions from mobile communication systems, the international agency for research on cancer (IARC) has concluded that EM radiation is possibly carcinogenic and categorized it as Group 2B - a

Y. A. Sambo is with the Department of Telecommunications Engineering, Baze University, Abuja, Nigeria. (Email: y.sambo@bazeuniversity.edu.ng). F. Héliot is with the Institute for Communication Systems (ICS), home of the 5G Innovation Centre, University of Surrey, Guildford, GU2 7XH, United Kingdom. (Email: f.heliot@surrey.ac.uk). M. A. Imran is with the School of Engineering, University of Glasgow, Glasgow, G12 8QQ, United Kingdom. (Email: muhammad.imran@glasgow.ac.uk). group reserved for systems that have limited evidence of carcinogenicity in humans [1]. Additionally, it has been reported in [2] that heavy users of wireless phones, over a period of more than 25 years, are three times more likely to develop a brain tumour. Consequently, the European Environmental Agency (EEA) has recommended precautionary approaches to minimize the effects of exposure to EM emissions such as the use of hands-free, limiting the usage of mobile phones (especially among children) and minimizing the duration of mobile phone calls, among others.

Even though there is a strong case for developing technical solutions to minimize EM emissions from mobile communication systems, very few research works on EM emission reduction have been carried out in such systems, as most researchers and equipment manufacturers have focused on operating within the regulatory prescribed limits. However, these limitations are designed for short-term usage and, hence, do not protect well enough from the long-term effects of EM exposure, as it has been argued in [2]. A new opportunity for research on EM emission in wireless communication systems presents itself with the advent of the $5 \mathrm{G}$ mobile system. It is envisioned that latency and EM emission, alongside traditional criteria such as spectral efficiency (SE) and EE, will play a key role in the design of the 5G system [3].

We have performed, in [4], a comprehensive survey of existing literature, dosimetry, metrics, international projects as well as guidelines and limits on exposure to EM emissions from mobile communication systems. We also reviewed and proposed several potential techniques for reducing EM emission in mobile systems. It has been shown in [5]-[7] that specific absorption rate (SAR) shielding, i.e., the use of ferrite materials or metamaterials between the mobile phone and the user's head, can minimize the EM emission in the uplink. However, these works mainly focus on reducing the SAR without considering the quality of service $(\mathrm{QoS})$ of the network. Given that uplink EM emission is directly related to the amount of energy (power over time) radiated towards a user, it suggests that 3-dimensional resource allocation (power, time and frequency) is well-suited for minimizing EM emission, while maintaining a desired QoS [8]. Thus, in [9], we proposed a user scheduling algorithm to minimize the EM emission of users in the uplink of TDMA, which reduces the EM emission by up to $45 \%$ in comparison with traditional TDMA system. In the single cell scenario, subcarrier and power allocation in the uplink of OFDM systems have been very well investigated but mainly for SE improvement [10], [11] and, recently, EE [12], [13]. In the context of EM emission reduction, in [14], we proposed a scheduler for the uplink of OFDM systems to minimize EM emissions and maintain QoS, based on the 
assumption that the channel state information (CSI) of all the users can be predicted over a certain transmission window (i.e., spanning several time slots). Our scheme takes into account the signaling power of all the users in the network and data transmission is confined to happen within the transmission window. The results show that spreading data transmission over a longer window makes the scheme to be very robust against the effect of imperfect channel prediction. For multicell systems, the authors in [15] and [16] have proposed scheduling and resource allocation algorithms for maximizing the SE in the uplink of OFDM systems. In Parallel, the authors in [17] and [18] have shown the benefits of coordination for mitigating interference and improving the EE in the downlink of OFDM systems. Accordingly, we have reported in [4] that (BS) coordination could also be effective to minimize EM emission in multicell systems. Thus, as a preliminary work on this topic, we have proposed in [19], an iterative scheme to minimize EM emission in the uplink of multicell OFDM systems, based on the assumption that each BS can iteratively optimize the transmissions of its users while maintaining QoS. From an EE perspective, more gain can be achieved by tuning the power at the BS, and this explains why EE-based coordination techniques have mainly been developed for the downlink. On the contrary, for EM emission, the uplink is where most of the reduction can be made, which motivates our work that provides a bespoke solution for the uplink.

In this paper, we propose a novel EM-aware scheduling (i.e., 3-dimensional resource allocation) scheme for the uplink of a coordinated multiuser OFDM wireless communication system. Contrary to [15], [16] that have been designed for optimal SE and do not take into account the QoS target, our proposed scheduler design takes into account the QoS, signaling power as well as the data transmission power of each user in the network to minimize the EM emission in the uplink of multicell OFDM systems. Our scheme minimizes the emitted energy subject to transmitting a target number of bits (QoS target) over a given transmission window, while taking into account the power constraint in each of the several time slots (TSs). This scheme is based on the assumption that long-term CSI of all the users in the network is available. Given that the original optimization problem is non-convex, we first reformulate it in a standard convex form and solve it by designing a waterfilling algorithm. We propose two power allocation algorithms for our scheme to minimize EM emission. Our first power allocation algorithm performs multicell iterative optimization to update the transmit powers of the users of different sectors, while our second power allocation algorithm uses the average channel gains of the users of different sectors to obtain an approximation of the transmit power without multicell iterative optimization. However, given that this approach only provides an approximation of the users' transmit power, some users may not always meet their target number of bits with this approach. Consequently, it must be refined by using it as a starting point of our first power allocation approach so that the target number of bits constraint is always met. This combined approach yields the same EM emission performance as the first power allocation algorithm on its own, but with a faster convergence rate i.e. lower computational complexity. The main contributions of this paper are listed as follows:

- Our proposed EM emission minimization scheme takes into account the QoS, signaling power as well as the data transmission power of each user to minimize the sum EM emission of users in the uplink of multicell OFDM systems.

- We extend our multicell EM emission-aware scheme of [19] by proposing a new subcarrier allocation algorithm that groups the users of different sectors by using the received interference power of each user at the neighboring BSs. We also propose a novel power allocation algorithm to minimize EM emission by using the average channel gains of the users of different sectors to obtain the approximate bit and power allocation of each individual user. This approach simplifies the optimization process as it provides a good starting point for optimizing the system.

- Simulation results demonstrate that our proposed scheme significantly outperforms the single cell EM emission minimization scheme of [14] (i.e., without intercell interference) by up to $70 \%$, and by over 3 and 4 orders of magnitude when compared to the multicell SE maximization scheme of [16] and the single cell SE maximization scheme of [11], respectively. Furthermore, the results show that the uplink EM emission in the network is proportional to the number of users and their QoS requirements.

The rest of this paper is organized as follows. Section II describes the system model for the uplink of a coordinated OFDM network as well as the relationship between the EM emission and the emitted energy. In Section III, we formulate and solve our EM emission-aware problem. In Section IV, the complexity analysis of our proposed scheme is presented. We analyze the numerical results in Section $\mathrm{V}$ and, finally, conclude the paper in Section VI.

\section{System MOdEL}

Consider the uplink of a classic planar multiuser OFDM cellular system, as depicted in Fig. 1, where sectorized singleantenna BSs communicate with $K$ uniformly distributed single antenna users per sector. In this setting, each user is surrounded by three BSs, i.e., its own serving BS and its two closest neighboring BSs. The system utilizes a total bandwidth $W$ divided into $N$ equal subcarriers. We assume that time is split into TSs, each of length $l$. Additionally, it is assumed that a central scheduler, which has perfect CSI of all the users in the network up to $T$ TSs in advance, coordinates the transmission. This can be achieved by using the uplink pilot signals that each user transmits to its serving BS; then the BSs forward this information to the scheduler which uses it to predict the channel gains of each user in the network for a window of $T$ TSs and, subsequently, perform subcarrier allocation. Note that predicting the CSI of up to $T$ TSs can be regarded as a theoretical assumption, however in practice, this approach is well-suited for quasi-static channels over $T$ TSs. For channels having larger variations over time, schemes like the ones in [20] and [21] can be used for CSI prediction. In this paper, a 


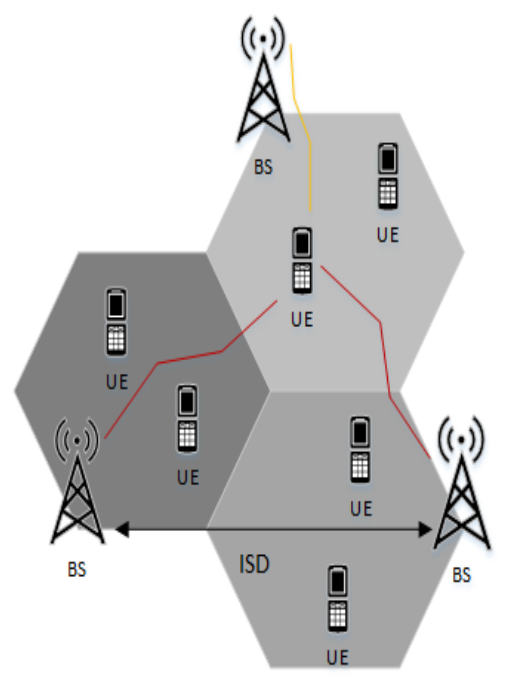

Figure 1. Sectorized cellular system model.

subcarrier can be allocated to at most one user per sector in a TS but a user can have more than one subcarrier in a TS. Hence, the amount of bits transmitted in a TS by user $k$ in sector $m$ can be expressed as

$$
b_{k}^{m}(t)=w l \sum_{n=1}^{N} \alpha_{k, n}^{m}(t) \log _{2}\left(1+\frac{p_{k, n}^{m}(t) g_{k, n}^{m}(t)}{\sigma^{2}+I_{k, n}^{m}(t)}\right),
$$

where $w$ denotes the bandwidth of a subcarrier, $p_{k, n}^{m}(t)$ and $g_{k, n}^{m}(t)$ represent the transmit power and channel gain, respectively, of user $k$ on subcarrier $n$ in sector $m$ at TS $t$. The parameter $\alpha_{k, n}^{m}(t)$ represents the subcarrier allocation index of user $k$, such that $\alpha_{k, n}^{m}(t)=1$ if subcarrier $n$ is allocated to user $k$ in sector $m$ at TS $t$ and $\alpha_{k, n}^{m}(t)=0$ otherwise, while $\sigma^{2}$ denotes the noise power per subcarrier. Additionally, $I_{k, n}^{m}(t)$ accounts for the interference from users transmitting on subcarrier $n$ at TS $t$ in the neighboring sectors such that

$$
I_{k, n}^{m}(t)=\sum_{v=1, v \neq m}^{M} p_{k, n}^{v}(t) g_{k(v), n}^{m}(t)
$$

where $g_{k(v), n}^{m}(t)$ denotes the channel gain of user $k$ on subcarrier $n$ in sector $v$ to the BS in sector $m$, while $M$ represents the number of sectors in the system.

From [22], the total uplink EM exposure of the system can be expressed as

$$
E=\sum_{m}^{M} \sum_{k}^{K} E_{k}^{m}
$$

where $E_{k}^{m}$ denotes the contribution of user $k$ in sector $m$ to the EM exposure index, and is given by

$$
E_{k}^{m}=\frac{\mathrm{SAR}_{k}^{m}}{P^{\mathrm{ref}}} \sum_{t}\left(\hat{p}_{k}^{m}(T)+\sum_{n} p_{k, n}^{m}(t)\right) l .
$$

In (4), $\mathrm{SAR}_{k}^{m}$ is the whole body averaged SAR of the $k$-th user mobile device while $P^{\text {ref }}$ and $\hat{p}_{k}^{m}(T)$ represent the incident reference power and the signaling power of user $k$ in sector $m$, respectively. In this paper, we assume that all the users in the system have similar devices, as well as $\mathrm{SAR}_{k}^{m}$ and $P^{\text {ref }}$, accordingly. Moreover, the signaling power, $\hat{p}_{k}^{m}(T)$, can be computed as [23]

$$
\hat{p}_{k}^{m}(T)=\min \left(P_{\max }, P_{0}+D_{k}^{m}+\Delta(T)\right)[\mathrm{dBm}],
$$

where $P_{0}$ denotes the received signal power threshold at the $\mathrm{BS}, D_{k}^{m}$ represents the path loss of user $k$ in sector $m$, and

$$
\Delta(T)= \begin{cases}10 \log _{10}(\delta / 4), & \text { if } \delta \geq 4 \\ 0, & \text { otherwise }\end{cases}
$$

such that $\delta$ denotes the number of bits that are transmitted for obtaining channel quality indication (CQI). In this paper, we assume that $a$ bits of CQI are transmitted by each user in a TS, hence $\delta=a T$ in (6), where $T$ denotes the number of TSs [23]. Hence, the number of signaling information bits that are transmitted increases with the size of the transmission window.

It can be remarked from (4) that if $\mathrm{SAR}_{k}^{m} / P^{\text {ref }}$ is similar for all the users, then reducing the EM exposure of any user in any sector, $E_{k}^{m}$, boils down to reducing its transmitted/emitted energy i.e., the product of the transmit power and time.

Consequently, our EM emission reduction scheme aims at reducing the total emitted energy in the system, based on the following optimization problem

$$
\min _{\mathbf{p}, \boldsymbol{\alpha}} \mathcal{E}(\mathbf{p}, \boldsymbol{\alpha})=\sum_{m}^{M} \sum_{k}^{K} l\left(\hat{p}_{k}^{m}(T)+\sum_{t=1}^{T} \sum_{n=1}^{N} \alpha_{k, n}^{m}(t) p_{k, n}^{m}(t)\right)
$$

subject to

$$
\begin{array}{r}
w l \sum_{t=1}^{T} \sum_{n=1}^{N} \alpha_{k, n}^{m}(t) \log _{2}\left(1+\frac{p_{k, n}^{m}(t) g_{k, n}^{m}(t)}{\sigma^{2}+I_{k, n}^{m}(t)}\right)=B_{k}^{m}, \\
\sum_{n=1}^{N} \alpha_{k, n}^{m}(t) p_{k, n}^{m}(t) \leq P_{k}^{\max } \forall t, \\
\sum_{k=1}^{K} \alpha_{k, n}^{m}(t) \leq 1 .
\end{array}
$$

In (7) - (8), $\mathcal{E}(\mathbf{p}, \boldsymbol{\alpha})$ denotes the total energy emitted by each user $k$ in sector $m$ to transmit $B_{k}$ bits, $B_{k}$ is a per-user target number of transmitted bit (QoS requirement) and $P_{k}^{\max }$ represents the maximum transmit power of the user equipment. Finally, $\mathbf{p}=\left[p_{1,1}^{1}(1), \ldots, p_{K, N}^{1}(T), p_{1,1}^{2}(1), \ldots, p_{K, N}^{M}(T)\right] \succeq$ 0 and $\boldsymbol{\alpha}=\left[\alpha_{1,1}^{1}(1), \ldots, \alpha_{K, N}^{1}(T), \alpha_{1,1}^{2}(1), \ldots, \alpha_{K, N}^{M}(T)\right] \in$ $\{0,1\}^{M K N T}$. Notice that the constraint (8a) on the number of transmitted bits is set across the whole $T$ TSs for all the users while the power constraint (8b) is per TS, as transmissions are performed in a slotted manner in time domain. 


\section{EM EMISSION REDUCTION SCHEME}

In this section, we propose an enhanced EM emission reduction algorithm for solving the problem in (7) - (8). In our scheme, the scheduler performs subcarrier allocation and power allocation across the whole $T$ TSs to minimize the transmission energy and, hence, EM emission.

It is worth mentioning that the problem in (7) - (8) is nonconvex due to the binary nature of $\alpha_{k, n}^{m}(t)$ such that this problem is NP-hard and, hence, intractable for large systems. A sub-optimal, but practical, way of simplifying this problem is to perform subcarrier and power allocations in a sequential manner as, for instance, in [11] or [12]; at first subcarrier allocation is performed to obtain $\alpha_{k, n}^{m}(t)$ values, then powers are allocated based on the knowledge of $\alpha_{k, n}^{m}(t) \forall k, n, t, m$.

\section{A. Subcarrier Allocation}

Unlike in single cell scheduling where a subcarrier is allocated to at most one user in the system, in the multicell scenario, users in different sectors can share the same subcarrier. This results in intercell interference that degrades system performance. Thus, in multicell systems, the central scheduler must group users of different sectors first and then assign subcarriers to each of these groups, as in our preliminary work of [19]. In this paper, we propose a novel approach for grouping the users of different sectors prior to subcarrier assignment; in our approach $K$ groups consisting of one user from each sector are formed based on the power of the pilots transmitted by each user received in neighboring sectors, given as

$$
\eta_{k}^{m}=\hat{p}_{k}^{m}(T) \sum_{q \neq m}^{M} D_{k(m)}^{q},
$$

where $D_{k(m)}^{q}$ denotes the path-loss from user $k$ in sector $m$ to neighboring BS $q$. Our new user grouping is simple and practical given that $\eta_{k}^{m}$ can easily be measured by each neighboring sector $q$ [24]. From an implementation point-ofview, we first compute $\eta_{k}^{m}$ for each user in each sector and then rank the users of each sector in increasing order of $\eta_{k}^{m}$, i.e., the user in sector $m$ with the least $\eta_{k}^{m}$ is ranked as 1 and the user with the highest $\eta_{k}^{m}$ is ranked as $K$. We then group the users of different sectors that have the same rank together. This approach ensures that the users that generate the least interference to neighboring sectors are grouped together. Let us define the user group index, $u_{j}$, for $j=1, \ldots, K$, such that each index represents one of the $K$ groups of $M$ users. We then treat the $u_{j}$-th group of users as a user, $j$, and perform subcarrier allocation for each user $j$ to minimize its EM emission by using its average channel gains, which greatly simplifies the subcarrier allocation. Our subcarrier allocation metric is defined as

$$
\Gamma_{j, n}(t)=\frac{h_{j, n}(t)}{\bar{h}_{j}},
$$

where $h_{j, n}(t)=g_{j, n}(t) / G_{j, n}(t)$, such that $g_{j, n}(t)=$ $\frac{1}{M} \sum_{m=1}^{M} g_{u_{j(m)}, n}^{m}(t), G_{j, n}(t)=\frac{1}{M} \sum_{m=1}^{M} G_{u_{j(m)}, n}^{m}(t)$ and $G_{u_{j(m)}, n}^{m}(t)=\sum_{\substack{v=1 \\ v \neq m}}^{M} g_{j(v), n}^{v}(t)$. Here, $u_{j(m)}$ refers to the user belonging to sector $m$ in the user group with index $u_{j}$.
The parameter $\Gamma_{j, n}(t)$ represents the unit power SIR of user $j$ on subcarrier $n$ at TS $t$ and $\bar{h}_{j}=\frac{\sum_{t}^{T} \sum_{n}^{N} h_{j, n}(t)}{N T}$ denotes the average SIR of user $j$ within the transmission window $T$ across the whole subcarriers, which is based on the CSI knowledge of all the users up to $T$ TSs. We assume here that this knowledge is available to compute $\Gamma_{j, n}(t), \forall j, n, t$ and order the subcarriers into a $K \times N T$ matrix with elements $\Gamma_{j, n}(t), \forall j, n, t$. Given that the channel gains of some users in a user group might be very good while those of other users might be very poor, averaging the channel gains of the users of each group means that the users with good channel gains will generate less interference to the users with worse channel gains, and vice versa, which would even out the effect of highly contrasting channel gains.

The subcarrier ordering is done in ascending order of the worst $\Gamma_{j, n}(t)$ on each subcarrier, as performed in [14]. Starting from the first column, the user with the best $\Gamma_{j, n}(t)$ on that subcarrier is allocated to it and then we move to the next column until $S$ subcarriers are allocated to each user, where $S=\lfloor N T / K\rfloor$ and $\lfloor$.$\rfloor denotes the floor operator. This$ approach maximizes both the $\min _{n \in \mathcal{N}_{j}}\left\{\Gamma_{j, n}\right\}$ and the average SIR value in $\mathcal{N}_{j}, \forall j$. Here, $\mathcal{N}_{j}$ denotes the set of subcarriers allocated to user $j$ within the transmission window, $T$. The users of each group $u_{j}$ are made to share the same set of subcarriers allocated to that user group, i.e., user $j$.

A practical way to obtain the subcarrier allocation metric, $\Gamma_{j, n}(t)$, is to start from the first user group index i.e., $j=1$, and to perform equal power allocation on the subcarriers of each user in that group, i.e. $p_{k, n}^{m}(t)=P / N, \forall k, n, m, t$. By using the values of $p_{k, n}^{m}(t), G_{u_{j(u)}, n}^{m}(t)$ can be obtained based on $p_{k, n}^{m}(t)$ and the received interference, $I_{k, n}^{m}(t)$, at each sector. This process is repeated for $j=2, \ldots, K$ to obtain $\Gamma_{j, n}(t)$ for the remaining user groups.

The subcarrier allocation phase of our proposed scheme involves choosing the worst channel gain on each subcarrier and sorting of all $N T$ subcarriers in ascending order of their worst $\Gamma_{j, n}$. This phase has a complexity of $O(N T(\log N T+K))$. The user-subcarrier pairing is a two dimensional search having a complexity of $O(K N T)$. Hence, the subcarrier allocation phase has a computational complexity of $O(N T(\log N T+$ $K))$.

\section{B. Power Allocation}

In this subsection, we propose two power allocation algorithms to minimize EM emission in the uplink of multicell systems. In our first power allocation algorithm (PA1), each sector minimizes the uplink EM emission by iteratively optimizing the transmit power of its users, while taking into account interference from neighboring sectors. Whereas our second power allocation algorithm (PA2) uses the average channel gains of the users of different sectors to obtain the approximate power allocation for each user.

1) Power allocation algorithm 1 (PA1): For any given subcarrier allocation, i.e., for fixed values of $\alpha_{k, n}^{m}(t)$, the power allocation problem is equivalent to $M K$ independent power allocation problems, one for each user of every sector. However, the optimization problem in (7) - (8) is still not 
convex, even for a fixed subcarrier allocation because of both the interference term and equality constraint in (8a); the latter being not affine [25]. Given that the transmit power of user $k$ on subcarrier $n$ in sector $m$ at TS $t$ can be expressed from (1) as

$$
p_{k, n}^{m}(t)=\frac{\left(2^{r_{k, n}^{m}(t)}-1\right) g_{k, n}^{m}(t)}{\sigma^{2}+I_{k, n}^{m}(t)},
$$

where $r_{k, n}^{m}(t)=b_{k, n}^{m}(t) / w l$ denotes the rate of user $k$ on subcarrier $n$ in sector $m$ at TS $t$. We can re-write (7) - (8) into a standard convex optimization format for given $I_{k, n}^{m}(t)$ and $\alpha_{k, n}^{m}(t)$ values. Hence, the power allocation problem of user $k$ in sector $m$ can be expressed as

$$
\begin{aligned}
& \min _{r_{k, n}^{m}(t)} \mathcal{E}_{k}^{m}= \\
& \hat{p}_{k}^{m} l+l \sum_{t=1}^{T} \sum_{n=1}^{N} \frac{\alpha_{k, n}^{m}(t)\left(2^{r_{k, n}^{m}(t)}-1\right)\left(\sigma^{2}+I_{k, n}^{m}(t)\right)}{g_{k, n}^{m}(t)}
\end{aligned}
$$

subject to

$$
\begin{aligned}
& w l \sum_{t=1}^{T} \sum_{n=1}^{N} \alpha_{k, n}^{m}(t) r_{k, n}^{m}(t)=B_{k}^{m} \\
& \sum_{n=1}^{N} \alpha_{k, n}^{m}(t)\left(2^{r_{k, n}^{m}(t)}-1\right)\left(\sigma^{2}+I_{k, n}^{m}(t)\right) / g_{k, n}^{m}(t) \leq P_{k}^{\max } .
\end{aligned}
$$

With the equality constraint being converted into an affine function, the problem in (12) with constrains (13a) and (13b) is convex in $r_{k, n}^{m}(t)$. This comes down to a rate allocation problem over all the subcarriers allocated to user $k$ in sector $m$ during the transmission window $T$. The Lagrangian of the problem can be defined as

$$
\begin{aligned}
& \mathcal{L}\left(r_{k}^{m}(t), \lambda_{k}^{m}, \mu_{k}^{m}(t)\right)= \\
& \hat{p}_{k}^{m} l+l \sum_{t=1}^{T} \sum_{n=1}^{N} \frac{\alpha_{k, n}^{m}(t)\left(2^{r_{k, n}^{m}(t)}-1\right)\left(\sigma^{2}+I_{k, n}^{m}(t)\right)}{g_{k, n}^{m}(t)} \\
& +\lambda_{k}^{m}\left(B_{k}^{m}-w l \sum_{t=1}^{T} \sum_{n=1}^{N} \alpha_{k, n}^{m}(t) r_{k, n}^{m}(t)\right)+\mu_{k}^{m}(t)\left(P_{k}^{\max }\right. \\
& \left.-\sum_{n=1}^{N} \alpha_{k, n}^{m}(t)\left(2^{r_{k, n}^{m}(t)}-1\right)\left(\sigma^{2}+I_{k, n}^{m}(t)\right) / g_{k, n}^{m}(t)\right),
\end{aligned}
$$

where $\lambda_{k}^{m}$ and $\mu_{k}^{m}(t)$ denote the Lagrange multipliers associated with the constraints (13a) and (13b), respectively. Given that the optimization problem in (12) - (13) is convex, the following Karush-Kuhn-Tucker (KKT) conditions [26] are necessary and sufficient conditions for optimality

$$
\begin{gathered}
w l \sum_{t=1}^{T} \sum_{n=1}^{N} \alpha_{k, n}^{m}(t) r_{k, n}^{m}(t)-B_{k}^{m}=0, \quad \forall k, m, \\
\sum_{n=1}^{N} \alpha_{k, n}^{m}(t)\left(2^{r_{k, n}^{m}(t)}-1\right)\left(\sigma^{2}+I_{k, n}^{m}(t)\right) / g_{k, n}^{m}(t)-P_{k}^{\max } \leq 0,
\end{gathered}
$$

$$
\begin{gathered}
\mu_{k}^{m}(t)\left(\sum_{n=1}^{N} \alpha_{k, n}^{m}(t)\left(2^{r_{k, n}^{m}(t)}-1\right)\left(\sigma^{2}+I_{k, n}^{m}(t)\right) / g_{k, n}^{m}(t)\right. \\
\left.-P_{k}^{\max }\right)=0, \forall k, m, t \\
\mu_{k}^{m}(t) \geq 0, \quad \forall k, m, t \\
\nabla \mathcal{L}\left(r_{k}^{m}, \lambda_{k}^{m}, \mu_{k}^{m}(t)\right)=0, \quad \forall k, m .
\end{gathered}
$$

By solving (19), we obtain the optimal solution to the problem in (12) - (13) as

$$
r_{k, n}^{m \star}(t)=\left[\log _{2} \nu+\log _{2}\left(\frac{w g_{k, n}^{m}(t)}{\ln (2)\left(\sigma^{2}+I_{k, n}^{m}(t)\right)}\right)\right]_{+},
$$

where $\nu$ is expressed as

$$
\nu=\frac{\lambda_{k}^{m \star}}{\left(1-\mu_{k}^{m \star}(t) / l\right)},
$$

and $[x]_{+}=\max \{x, 0\}$. Note that $(20)$ is a rate-based waterfilling solution, with $\nu$ denoting the water level. Several iterative algorithms like Secant and Newton-Raphson methods [27] can be used to obtain the optimal values $\lambda_{k}^{m \star}$ and $\mu_{k}^{m \star}(t)$ by fixing one of them and iteratively finding the other one until convergence. The variables $\lambda_{k}^{m \star}$ and $\mu_{k}^{m \star}(t)$ have to satisfy the constraints (12) and (13), respectively. Knowing $r_{k, n}^{m \star}(t) \forall k, n, m, t$, the optimal per-subcarrier transmit powers can then be obtained from (11).

The bit allocation phase of our PA1 algorithm involves each sector obtaining the Lagrange multipliers by using the secant method and it has a complexity of $O\left(\theta N T\left(\theta_{1}+\theta_{2}\right)\right)$, where $\theta_{1}$ and $\theta_{2}$ denote the number of iterations it takes to obtain $\lambda_{k}^{m \star}$ and $\mu_{k}^{m \star}(t)$, respectively, while $\theta$ represents the number of iterations it takes for the rate allocation process to converge. However, given that this approach involves multicell iteration to minimize the transmit power, the complexity of PA1 is $O\left(\psi M \theta\left(\theta_{1}+\theta_{2}\right)\right)$, where $\psi$ denotes the number of iterations it takes for the system to converge. Thus, our proposed scheme with PA1 has a complexity of $O\left(N T\left(\log N T+K+\psi M \theta\left(\theta_{1}+\right.\right.\right.$ $\left.\left.\theta_{2}\right)\right)$ ).

2) Power allocation algorithm 2 (PA2): By using the average channel gains of the users in each user group, $u_{j}$, the average number of bits transmitted by the $j$-th user in a $\mathrm{TS}$ is given as

$$
\tilde{b}_{j}(t)=w l \sum_{n=1}^{N} \alpha_{j, n}(t) \log _{2}\left(1+\frac{\tilde{p}_{j, n}(t) g_{j, n}(t)}{\sigma^{2}+\tilde{p}_{j, n}(t) G_{j, n}(t)}\right),
$$

where $\tilde{p}_{j, n}(t)$ denotes the transmit power of the $j$-th user (average transmit power of the users in $u_{j}$ ). Hence, the average transmit power of user $j$ on subcarrier $n$ at TS $t$ can be expressed from (22) as

$$
\tilde{p}_{j, n}(t)=\frac{\left(2^{\tilde{r}_{j, n}(t)}-1\right) g_{j, n}^{-1}(t) \sigma^{2}}{1-\left(2^{\tilde{r}_{j, n}(t)}-1\right) g_{j, n}^{-1}(t) G_{j, n}(t)},
$$

where $\tilde{r}_{j, n}(t)=\tilde{b}_{j, n}(t) / w l$ denotes the rate of user $j$ on subcarrier $n$ at TS $t$. Hence, the power allocation problem of user $j$ can be formulated as a function of $\tilde{r}_{j, n}(t)$ such that 


$$
\begin{aligned}
& \min _{r_{j, n}(t)} \tilde{\mathcal{E}}_{j}= \\
& \hat{p}_{j}(T) l+l \sum_{t=1}^{T} \sum_{n=1}^{N} \frac{\alpha_{j, n}(t)\left(2^{\tilde{r}_{j, n}(t)}-1\right) g_{j, n}^{-1}(t) \sigma^{2}}{1-\left(2^{\tilde{r}_{j, n}(t)}-1\right) g_{j, n}^{-1}(t) G_{j, n}(t)}
\end{aligned}
$$

subject to

$$
\begin{aligned}
& w l \sum_{t=1}^{T} \sum_{n=1}^{N} \alpha_{j, n}(t) \tilde{r}_{j, n}(t)=B_{j}, \\
& \sum_{n=1}^{N} \frac{\alpha_{j, n}(t)\left(2^{\tilde{r}_{j, n}(t)}-1\right) g_{j, n}^{-1}(t) \sigma^{2}}{1-\left(2^{\tilde{r}_{j, n}(t)}-1\right) g_{j, n}^{-1}(t) G_{j, n}(t)} \leq P_{j}^{\max } .
\end{aligned}
$$

The optimization problem is now clearly convex and its Lagrangian can be defined as

$$
\begin{aligned}
& \mathcal{L}\left(\tilde{r}_{j}(t), \lambda_{j}, \mu_{j}(t)\right)= \\
& \hat{p}_{j}(T) l+l \sum_{t=1}^{T} \sum_{n=1}^{N} \frac{\alpha_{j, n}(t)\left(2^{\tilde{r}_{j, n}(t)}-1\right) g_{j, n}^{-1}(t) \sigma^{2}}{1-\left(2^{\tilde{r}_{j, n}(t)}-1\right) g_{j, n}^{-1}(t) G_{j, n}(t)} \\
& +\lambda_{j}\left(B_{j}-w l \sum_{t=1}^{T} \sum_{n=1}^{N} \alpha_{j, n}(t) \tilde{r}_{j, n}(t)\right)+\mu_{j}(t)\left(P_{j}^{\max }\right. \\
& \left.-\sum_{n=1}^{N} \frac{\alpha_{j, n}(t)\left(2^{\tilde{r}_{j, n}(t)}-1\right) g_{j, n}^{-1}(t) \sigma^{2}}{1-\left(2^{\tilde{r}_{j, n}(t)}-1\right) g_{j, n}^{-1}(t) G_{j, n}(t)}\right)
\end{aligned}
$$

where $\lambda_{j}$ and $\mu_{j}(t)$ denote the Lagrange multipliers (slack variables) associated with the constraints (25a) and (25b), respectively. By applying the KKT conditions and solving $\nabla \mathcal{L}\left(\tilde{r}_{j}, \lambda_{j}, \mu_{j}(t)\right)=0$, we obtain the optimal solution to the problem in (24) - (25b) as

$$
\tilde{r}_{j, n}^{\star}(t)=\log _{2}\left(\frac{a+\beta+\sqrt{\beta(2 a+\beta)}}{2 \lambda_{j}^{\star} w l G_{j, n}^{2}(t)}\right)
$$

where

$$
\begin{gathered}
a=2 \lambda_{j}^{\star} w l G_{j, n}(t)\left(g_{j, n}(t)+G_{j, n}(t)\right), \\
\beta=\ln (2) g_{j, n}(t) \sigma^{2}\left(l-\mu_{j}^{\star}(t)\right) .
\end{gathered}
$$

The parameter $\tilde{r}_{j, n}^{\star}(t)$ denotes the optimal rate of the $j$-th user on subcarrier $n$ at TS $t$. The same root finding techniques described PA1 can be used to obtain $\lambda_{j}^{\star}$ and $\mu_{j}^{\star}(t)$. Knowing the optimal rate of each subcarrier allocated to user $j$ via (27), the optimal per-subcarrier transmit powers (according to our user grouping) that minimize the EM emission subject to transmitting $B_{j}$ bits can then be obtained from (23).

Note that the power allocation obtained is an approximation given that the average channel gains of each user group are used. Hence, (8a) might not always hold for the users of each group. To ensure that constraint (8a) is maintained for all the users, the approximate transmit powers obtained via PA2 are used as a starting point in PA1, and multicell iterative optimization is performed to satisfy (8a) and (8b).

Our PA2 algorithm has a complexity of $O\left(\tilde{\theta} N T\left(\tilde{\theta}_{1}+\tilde{\theta}_{2}\right)\right)$ for obtaining the approximate transmit power since it does not involve any multicell iteration, where $\tilde{\theta}, \tilde{\theta}_{1}$ and $\tilde{\theta}_{2}$ denote the number of iterations it takes to obtain $\tilde{r}_{j}^{\star}(t), \lambda_{j}^{\star}(t)$ and $\mu_{j}^{\star}(t)$, respectively. However, in order to ensure compliance with the target number of bits, multicell iterative power update would have to be performed. The complexity of PA2 with multicell iterative power update is $O\left(\Psi M \check{\theta}\left(\check{\theta}_{1}+\check{\theta}_{2}\right)\right)$, where $\Psi$ denotes the number of iterations it takes for the system to converge, while $\check{\theta}, \check{\theta}_{1}$ and $\check{\theta}_{2}$ denote the number of iterations it takes to obtain $r_{k, n}^{m \star}(t), \lambda_{k}^{m \star}$ and $\mu_{k}^{m \star}(t)$, respectively. Hence, the overall complexity of our proposed scheme with PA2 and multicell iteration is $O\left(N T\left(\log N T+K+\Psi M \check{\theta}\left(\check{\theta}_{1}+\check{\theta}_{2}\right)\right)\right)$.

\section{Scheduler Algorithm}

As shown in the flowchart in Fig. 2, the central scheduler first groups the users of different sectors together and then performs subcarrier allocation for the users of each group across all $M$ sectors. For PA1, each BS initially performs equal power allocation for each user on their respective subcarriers, i.e.

$$
p_{k, n}^{m}(t)= \begin{cases}\frac{\left|\mathcal{N}_{k}^{m}(t)\right|}{P_{k}^{\max },}, & \text { if } \alpha_{k, n}^{m}(t)=1 \\ 0, & \text { otherwise, }\end{cases}
$$

where $\left|\mathcal{N}_{k}^{m}(t)\right|$ denotes the number of subcarriers allocated to user $k$ in sector $m$ at TS $t$, and it is used as a starting point for the multicell optimization. In the next iteration, each BS measures the interference received for each of its served users from the initial power allocation and optimizes their transmissions to obtain the power allocation by using the interferences recorded in the previous iteration. This process is repeated until convergence is achieved.

As shown in Fig. 2, the approximate power allocation for each user group is first obtained. This power allocation is then fed into PA1 if the QoS is not met, i.e., (8a) is not satisfied. Note that the main difference between PA1 and PA2 is the usage of approximate transmit powers instead of equal transmit powers before computing the interference for the first time. This approach significantly reduces the computational complexity of our power allocation procedure, as it is next shown in Fig. 3.

It is worth noting that constraints (8a) and (8b) might not always hold concurrently for some values of $B, N, K$ and $T$. In order to ensure that both (8a) and (8b) are met, the transmission duration could be varied depending on the target number of bits. Indeed, we have shown in [14] that EM emission reduces as the transmission duration increases. This is because more subcarriers become available to the users and, as a result, a lower transmission power is needed to transmit the target number of bits.

Fig. 3 illustrates the convergence speed of our proposed power allocation algorithm. It can be seen that the value of the per-sector EM emission monotonically decreases after each iteration until convergence is achieved, which is consistent with a convex problem convergence behaviour. In this particular setting, where $B_{k}^{m}=20 \mathrm{kbit}, \forall k, m, K=10$ users, $I S D=700 \mathrm{~m}$ and $T=10 \mathrm{TSs}$, our PA1 algorithm requires, on average, 13 iterations to converge while our PA2 algorithm with multicell iterative power update (PA2 combined with PA1) requires only 7 iterations to converge, which represents a computational complexity reduction of about $50 \%$ in comparison with PA1. 


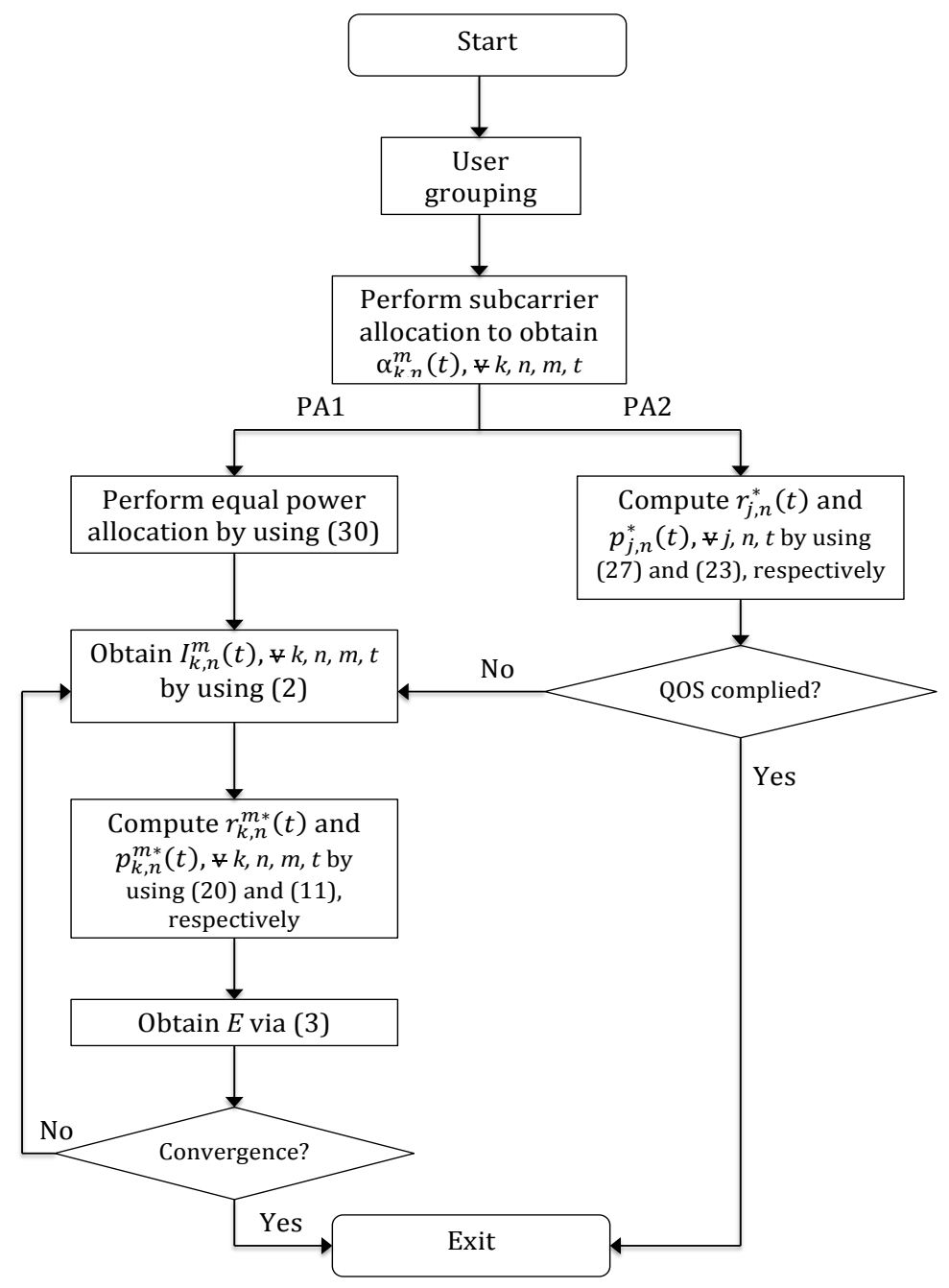

Figure 2. Flowchart of our proposed EM emission minimization scheme.

\section{NUMERICAL RESULTS AND DISCUSSIONS}

In this section, we compare the performance of our proposed EM emission reduction scheme against three existing schemes by using Monte Carlo simulation. We assume the $\mathrm{SAR}_{k}$ (measured in $\mathrm{W} / \mathrm{kg}$ ) and $P^{\text {ref }}$ (measured in $\mathrm{W}$ ) of all the users in the network to be the same and of unit values, i.e., $\mathrm{SAR}_{k} / P^{\mathrm{ref}}=\mathrm{SAR} / P^{\mathrm{ref}}=1, \forall k$. We further assume that all the users transmit the same target number of bits, i.e., $B_{k}^{m}=B \forall k, m$, while the fast fading and path-loss follow the model used in [14]. We compare our scheme against a state of the art uplink multicell (MC) SE maximization scheme of [16] and two single cell (SC) schemes, i.e., our EM emission reduction scheme of [14] and a classic SE maximization scheme [11], where each sector uses different frequency bands i.e., the number of subcarriers is split amongst the $M$ sectors, to avoid interference. In the following, the results of three different versions of our scheme are presented, where "proposedPA1" denotes the version of our proposed scheme using PA1 power allocation, while "proposed-PA2 scheme (approx.)" and "proposed-PA2 scheme with PU" represent the versions of our proposed scheme using PA2 with approximation and PA2 with multicell iterative power update, respectively. Both the multicell and single cell SE maximization schemes perform per TS optimization to avoid interference and improve SE of the network. Each user transmits signaling power in each TS as long as their target number of bits has not been met. Transmission for each user ceases as soon as the target number of bits has been met, in order to reduce the emitted energy. The numerical values of the parameters considered in our simulations are summarized in Table 1.

In Fig. 4, we compare the per-sector EM emission of our proposed scheme against the SC EM emission reduction scheme of [14], as well as the SE maximization schemes of [16] and [11], for $K=10$ users, $I S D=700 \mathrm{~m}$ and $T=10$ TSs. The target number of bits is varied from $10 \mathrm{kbit}$ to $20 \mathrm{kbit}$, representing a data rate of $1 \mathrm{Mbps}$ to $2 \mathrm{Mbps}$, respectively. It 


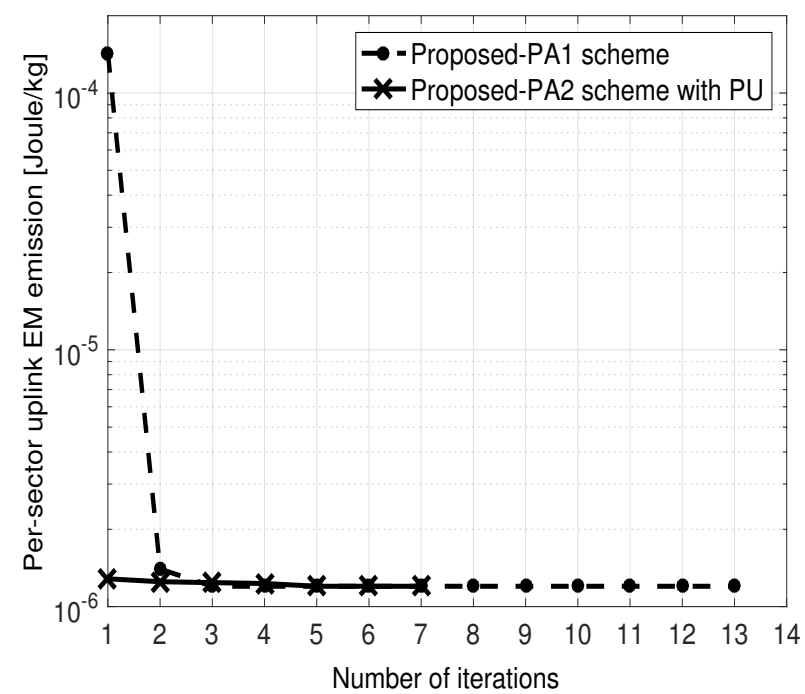

Figure 3. Convergence speed of our proposed EM emission reduction scheme for $B_{k}^{m}=20$ kbit, $\forall k, m, K=10$ users, $I S D=700 \mathrm{~m}$ and $T=10$ time slots.

can be observed that our proposed scheme produces the least EM emission of all the compared schemes, thus supporting the intuition that coordination, is indeed, beneficial towards minimizing EM emission in multicell systems. Furthermore, it can be remarked that the per-sector EM emission of all the schemes increases as the target number of bits increases. This is due to the increased power needed to achieve the higher target number of bits given that the number of subcarriers allocated to each user is fixed in all the EM emission reduction schemes; while in the SE maximization schemes, the users require more TSs to transmit their data and achieve the target. Increasing the number of TSs used for data transmission will result in using more signaling power for both the SE maximization schemes. Additionally, all the users will have to transmit signaling power as long as their target number of bits is not met in both SE maximization schemes. Our proposed scheme with PA2 (approximation) performs within $6 \%$ of our proposed scheme with PA1. This gives a good approximation of the transmission requirements for each user without the need for multicell iterative power update (PU). However, when compliance with the target number of bits is ensured, the performance of PA2 matches PA1. Additionally, our proposed scheme achieves up to $67 \%$ reduction in persector EM emission when compared with the SC EM emission reduction scheme of [14], and over 3 orders of magnitude, when compared to the two SE maximization schemes. The SC SE maximization scheme of [11] has at least 50\% higher EM emission when compared to the MC SE maximization scheme of [16]. This is because the SC schemes have fewer subcarriers in each cell, i.e., $N / M$ subcarriers, as well as the interference avoidance employed by the SE scheme of [16].

Fig. 5 depicts the per-sector EM emission of the same schemes as in Fig. 3, but versus the number of users in the network for a target of $B=10 \mathrm{kbit}, I S D=700 \mathrm{~m}$ and $T=10$ TSs. It is evident that the per-sector EM emission increases with the number of users in the network. This is
Table I

Simulation PARAMETERS

\begin{tabular}{|l|r|}
\hline Parameter & Value \\
\hline System Bandwidth $(W)$ & $20 \mathrm{MHz}$ \\
\hline Number of subcarriers $(N)$ & 256 \\
\hline Number of sectors $(\mathrm{M})$ & 3 \\
\hline Duration of 1 TS $(l)$ & $1 \mathrm{~ms}$ \\
\hline Rx signal Power threshold $\left(P_{0}\right)$ & $-112 \mathrm{dBm}$ \\
\hline Number of CQI bits $(a)$ & $4 \mathrm{bits}$ \\
\hline Max. User Tx Power $\left(P_{\max }\right)$ & $0.2 \mathrm{~W}$ \\
\hline SAR & $1 \mathrm{~W} / \mathrm{kg}$ \\
\hline$P^{\text {ref }}$ & $1 \mathrm{~W}$ \\
\hline Noise power density $\left(n_{0}\right)$ & $-174 \mathrm{dBm} / \mathrm{Hz}$ \\
\hline
\end{tabular}

because the number of subcarriers allocated to each user reduces as the number of users in the network increases since the users have to share a pre-defined (limited by the transmission window length) number of available subcarrier in EM emission reduction schemes. It implies that the users would have to transmit with more power to achieve their respective target number of bits. Whereas in both SE maximization schemes, more TSs are utilized to achieve the target number of bits for all the users in the network when the number of users increases. As in Fig. 4, our PA2 algorithm matches PA1 when the power allocation is fine-tuned to ensure QoS compliance. Furthermore, our proposed EM emission reduction scheme achieves an EM emission reduction of up to $70 \%$ when compared to the SC EM emission reduction scheme of [14] and by over 3 orders of magnitude when compared to the SE maximization schemes.

Fig. 6 shows the effect of increasing the inter-site distance (ISD) on the per-sector EM emission for $K=10$ users, $B=15$ kbit and $T=10$ TSs. It can be seen that EM emission increases as the ISD increases due to the effect of path-loss; users would have to transmit with higher power in order to mitigate it, which also leads to higher interference. As expected, our proposed scheme with PA2 matches the performance of our proposed scheme with PA1. It is evident that our proposed scheme reduces EM emission by up to $70 \%$ when compared to the EM emission reduction scheme of [14] and by over 3 to 4 orders of magnitude when compared to the SE maximization schemes.

In Fig. 7, we compare the per-sector EM emission of our proposed schemes against the benchmark schemes for the same bit-rates. The bit-rate of our proposed schemes was matched to those of the benchmark schemes by fixing the data transmission window to the same TSs as those used by the benchmark schemes. In the top plot of Fig. 7, we show a comparison between the proposed schemes versus the SC 


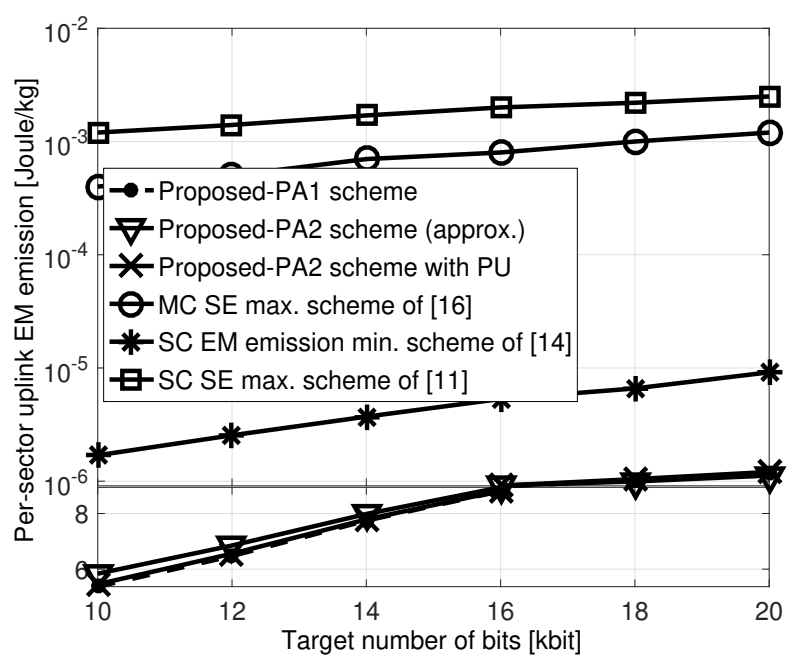

Figure 4. Per-sector EM emission comparison of our proposed EM emission reduction scheme versus the target number of bits for $K=10$ users, $I S D=$ $700 \mathrm{~m}$ and $T=10$ time slots.

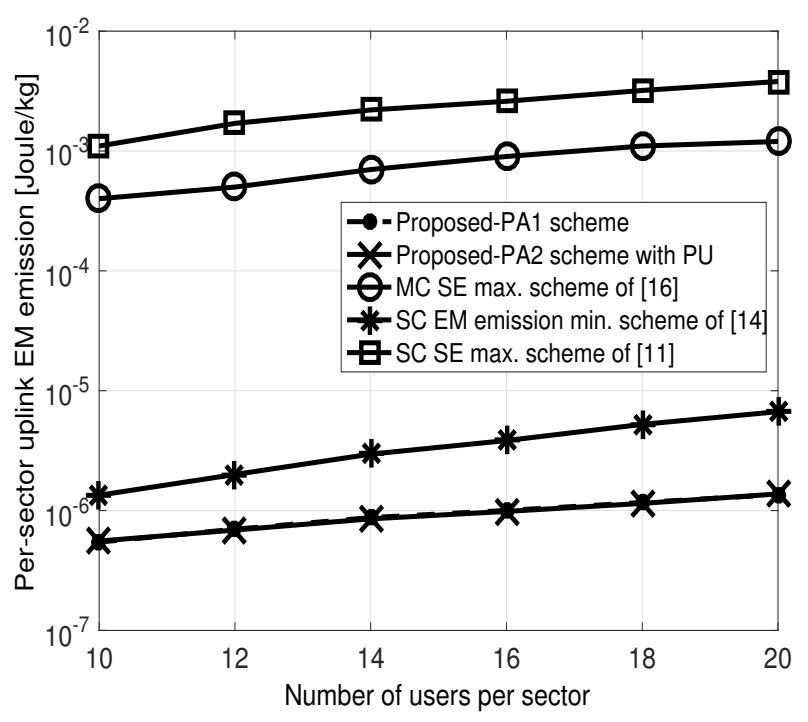

Figure 5. Per-sector EM emission comparison of our proposed EM emission reduction scheme versus the per-sector number of users for $B=10 \mathrm{kbit}$, $I S D=700 \mathrm{~m}$ and $T=10$ time slots.

SE max. scheme of [11]. The bit-rate of this plot varied from 0.549 Mbps to 0.9 Mbps. By matching the bit-rate of our proposed schemes to that of the benchmark scheme of [11], we show that our proposed schemes outperform the SC SE max. scheme of [11] by up to 3 orders of magnitude at low target number of bits. However, the performance gap begins to reduce as the target number of bits increases. This is because there is an increase in bit-rate as more bits would have to be transmitted within the fixed transmission window, which results in an increase in EM emission. The bottom plot of Fig. 7 shows the EM emission comparison of our proposed schemes versus the MC SE max. scheme of [16]. The bitrate of this plot varied from $0.495 \mathrm{Mbps}$ to $0.5 \mathrm{Mbps}$. It can be observed that our proposed schemes outperform the benchmark scheme of [16] by up to 3 orders of magnitude. The fluctuation in the performance of our proposed schemes is due to the change in data-rate of the benchmark MC SE

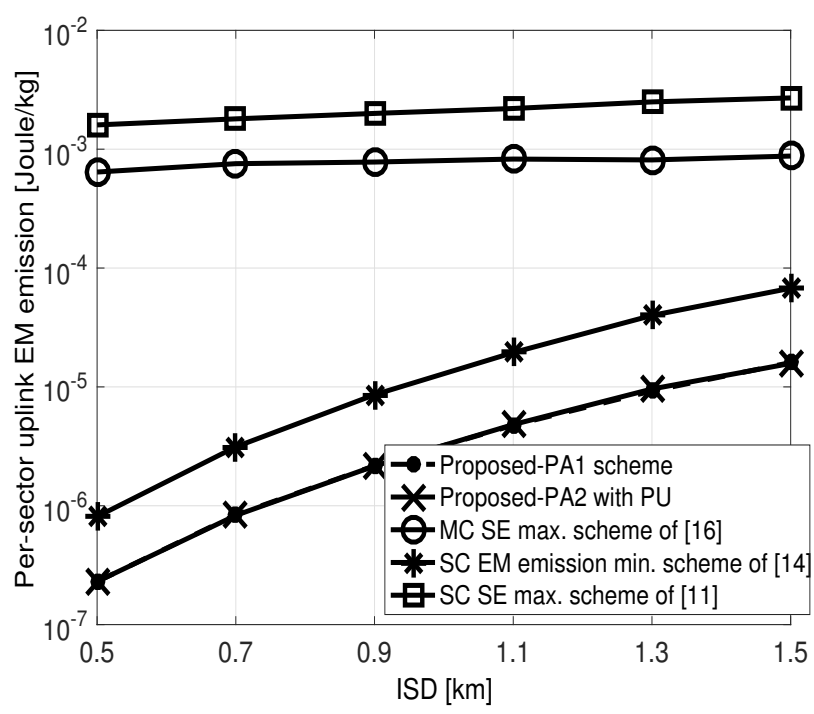

Figure 6. Per-sector EM emission comparison of our proposed EM emission reduction scheme versus the ISD for $B=15 \mathrm{kbit}, K=10$ users and $T=10$ time slots.

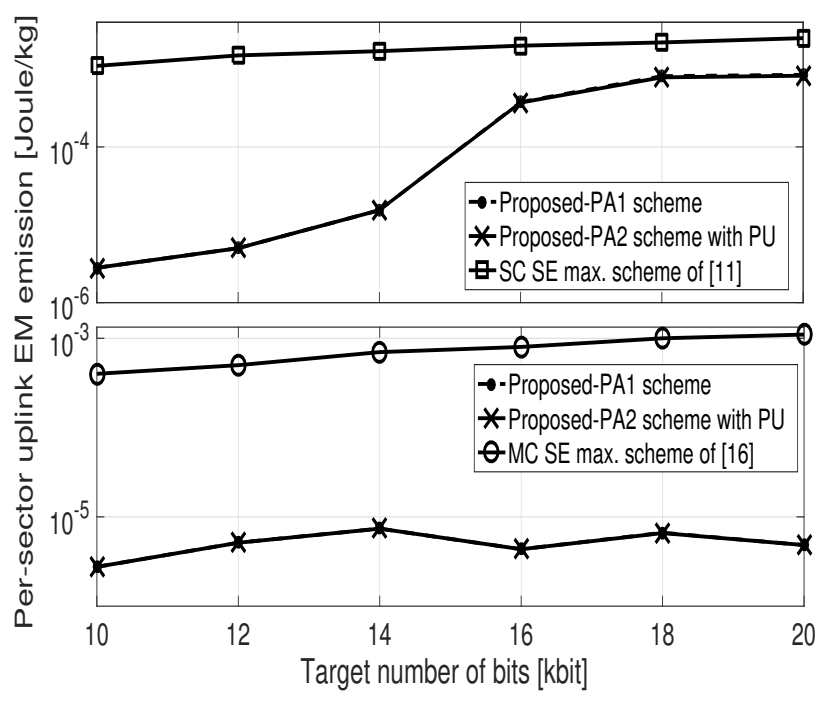

Figure 7. Per-sector EM emission comparison of our proposed schemes versus the target number of bits for $K=10$ users, $I S D=700 \mathrm{~m}$ and the same bit-rates.

max. scheme of [16], as the number of TSs needed to transmit each user's data increases. Fig. 7 confirms that our proposed schemes outperform the SE max. schemes of [11] and [16], even for the same bit-rates.

In Fig. 8, we show the relative computational complexity of the various schemes in Figs. 4, 5, 6 and 7 versus the target number of bits for $K=10$ users, $I S D=700 \mathrm{~m}$ and $T=10$ TSs. The relative complexity is defined as the ratio of the CPU execution time of any scheme to the CPU execution time of the SC SE maximization scheme of [11], which acts as a reference. It can be observed that the SC schemes have lower computational complexity when compared to the MC schemes. This is is due to the absence of interference, which means that these SC schemes do not require user grouping nor multicell iterative power allocation. Regarding the multicell scheme, our scheme complexity is roughly 1 order of magnitude more 


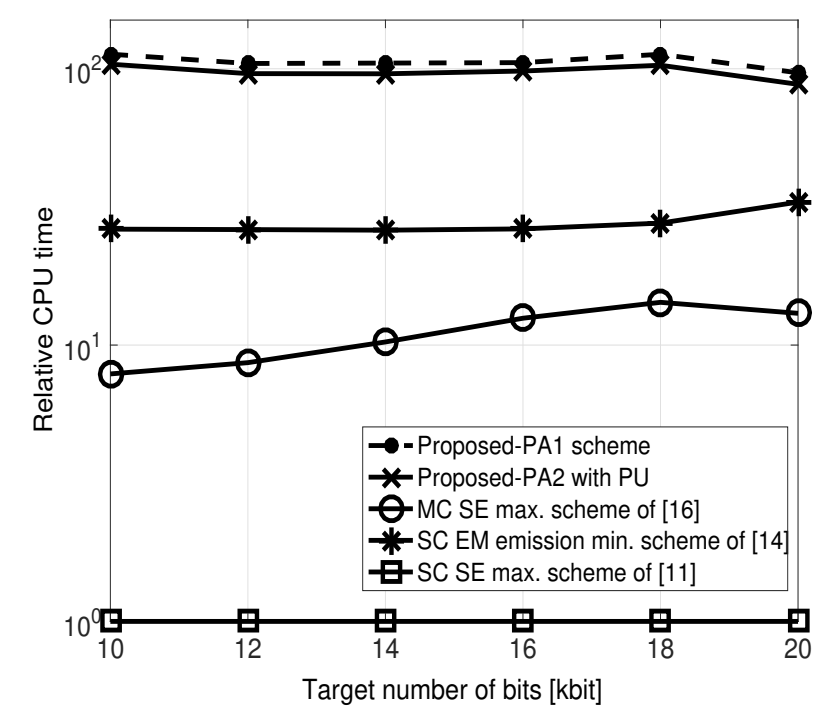

Figure 8. Relative CPU time complexity comparison versus the target number of bits for $K=10$ users, $I S D=700 \mathrm{~m}$ and $T=10$ time slots.

complex than the scheme of [16]. However, this is a fair price to pay for reducing the exposure by up to 3 order of magnitude. Furthermore, it can be seen that our proposed scheme with PA1 has about $10 \%$ higher complexity when compared to PA2 with multicell iterative power update.

\section{CONCLUSION}

In this paper, we have proposed a novel scheme for minimizing the EM emission of users in the uplink of multicell OFDM systems. The scheme is based on the assumption that the network can predict the long-term CSI of all the users in the network and then an optimal power allocation is performed to obtain the transmit power for each user. We also propose two power allocation algorithms to minimize EM emission. Our first power allocation algorithm performs multicell iterative power update to minimize EM emission by executing a ratebased water-filling algorithm to obtain the optimal rate and power allocation of all the users across all sectors. Conversely, our second power allocation algorithm is based on grouping the users of different sectors together and using their average channel gains to reduce the complexity of the power allocation. Consequently, the approximate transmit power of each user in the network that minimizes each user's EM emission is obtained. Simulation results show that our proposed scheme reduces EM emission by up to $70 \%$ when compared to the EM emission reduction scheme based on [14] and by up to 3 orders of magnitude when compared to the SE maximization schemes.

\section{ACKNOWLEDGMENT}

This paper reports work (partially) undertaken in the context of the project LEXNET. LEXNET is a project supported by the European Commission in the 7th Framework Programme (GA n $\left.{ }^{\circ} 318273\right)$. For further information, please visit www. lexnet-project.eu.

\section{REFERENCES}

[1] International Agency for Research on Cancer (IARC), "IARC Classifies Radiofrequency Electromagnetic Fields as Possibly Carcinogenic to Humans," Press Release No. 208, May 2011.

[2] L. Hardell and M. Carlberg, "Mobile phone and cordless phone use and the risk for glioma-analysis of pooled case-control studies in sweden, 1997-2003 and 2007-2009," http://dx.doi.org/10.1016/j.pathophys.2014. 10.001, 2014, Online; accessed Feb. 3, 2015.

[3] N. Cardona et. al, "Scientific Challenges Towards 5G Mobile Communications," COST IC1004 White paper, Tech. Rep., Dec. 2013.

[4] Y. A. Sambo, F. Héliot, and M. A. Imran, "A Survey and Tutorial of Electromagnetic Radiation and Reduction in Mobile Communication Systems," IEEE Commun. Surveys Tutorials, vol. 17, no. 2, pp. 790802,2015 .

[5] M. T. Islam, M. R. I. Faruque, and N. Misran, "Reduction of specific absorption rate (SAR) in the human head with ferrite material and metamaterial," Progress In Electromagnetics Research C, vol. 9, pp. 47-58, 2009.

[6] L. Ragha and M. Bhatia, "Evaluation of SAR reduction for mobile phones using RF shields," International Journal of Computer Applications, vol. 1, no. 13, pp. 80-85, Jan. 2010.

[7] R. Gómez-Villanueva, H. Jardon-Aguilar, and R. Linares y Miranda, "State of the art methods for low SAR antenna implementation," in Proc. 2010 Proceedings of the Fourth European Conference on Antennas and Propagation (EuCAP), Barcelona, Spain, Apr. 2010, pp. 1-4.

[8] M. Tesanovic et al., "The LEXNET project. wireless networks and EMF: Paving the way for low-emf networks," IEEE Vehicular Technology Magazine, vol. 6, no. 2, Jun. 2014.

[9] Y. A. Sambo, F. Héliot, and M. A. Imran, "A user scheduling scheme for reducing electromagnetic (EM) emission in the uplink of mobile communication systems," in Proc. Green Communications (OnlineGreencomm), 2014 IEEE Online Conference on, Online, Nov. 2014, pp. 1-5.

[10] K. Kim, Y. Han, and S. Kim, "Joint subcarrier and power allocation in uplink OFDMA systems," IEEE Commun. Lett., vol. 9, no. 6, pp. 526-528, Jun. 2005.

[11] M. Al-Imari, P. Xiao, M. A. Imran, and R. Tafazolli, "Low complexity subcarrier and power allocation algorithm for uplink OFDMA systems," EURASIP Journal on Wireless Commun. and Networking, vol. 98, no. 1, 2013.

[12] S. Buzzi, G. Colavolpe, D. Saturnino, and A. Zappone, "Potential games for energy-efficient power control and subcarrier allocation in uplink multicell OFDMA systems," IEEE Journal of Selected Topics in Signal Processing, vol. 6, no. 2, pp. 89-103, Apr. 2012.

[13] G. Miao, N. Himayat, G. Y. Li, and S. Talwar, "Low-complexity energy-efficient scheduling for uplink OFDMA," IEEE Transactions on Commun., vol. 60, no. 1, pp. 112-120, Jan. 2012.

[14] Y. A. Sambo, M. Al-Imari, F. Héliot, and M. A. Imran, "Electromagnetic Emission-aware Schedulers for the Uplink of OFDM Wireless Communication Systems," IEEE Transactions on Vehicular Technology, vol. PP, no. $99,2016$.

[15] H. Tabassum, Z. Dawy, and M. Alouini, "Sum rate maximization in the uplink of multi-cell OFDMA networks," in Proc. 7th International Wireless Commun. and Mobile Computing Conference (IWCMC), Istanbul, Turkey, Jul. 2011, pp. 1152-1157.

[16] E. Yaacoub and Z. Dawy, "Proportional fair scheduling with probabilistic interference avoidance in the uplink of multicell OFDMA systems," in Proc. IEEE GLOBECOM Workshops (GC Workshops), Miami, FL, Dec. 2010, pp. 1202-1206.

[17] F. Héliot, M. A. Imran, and R. Tafazolli, "Low-complexity energy efficient coordinated resource allocation in cellular systems," IEEE Transactions on Commun., vol. 61, no. 6, pp. 2271-2281, Jun. 2013.

[18] L. Venturino, A. Zappone, C. Risi, and S. Buzzi, "Energy-Efficient Scheduling and Power Allocation in Downlink OFDMA Networks With Base Station Coordination," IEEE Transactions on Wireless Commun., vol. 14, no. 1, pp. 1-14, Jan. 2015.

[19] Y. A. Sambo, F. Héliot, and M. A. Imran, "Electromagnetic emissionaware scheduling for the uplink of coordinated ofdm wireless systems," in Proc. 2015 IEEE Online Conference on Green Commun. (OnlineGreencomm), Piscataway, NJ, Nov. 2015, pp. 42-46.

[20] D. Schafhuber, G. Matz, and F. Hlawatsch, "Adaptive prediction of timevarying channels for coded OFDM systems," in Proc. IEEE International Conference on Acoustics, Speech, and Signal Processing (ICASSP), vol. 3, Orlando, FL, USA, may 2002, pp. 2549-2552.

[21] I. Wong, A. Forenza, R. Heath, and B. Evans, "Long range channel prediction for adaptive OFDM systems," in Proc. 38th Asilomar Conference on Signals, Systems and Computers, vol. 1, Nov. 2004, pp. 732-736. 
[22] N. Varsier et al., "D2.4: Global Wireless Exposure Metric Definition," INFSO-ICT-318273 LEXNET (Low EMF Exposure Future Networks), Tech. Rep., Nov. 2013, [Online]. Available: http://www.lexnet.fr/.

[23] 3GPP, "Evolved Universal Terrestrial Radio Access (E-UTRA); Physical Layer Procedures," 3rd Generation Partnership Project (3GPP), TS 36.213 V10.3.0, 2011.

[24] X. Li, E. Bjornson, E. G. Larsson, S. Zhou, and J. Wang, "Massive MIMO with multi-cell MMSE processing: Exploiting all pilots for interference suppression," CoRR, vol. abs/1505.03682, 2015. [Online].
Available: http://arxiv.org/abs/1505.03682

[25] Z.-Q. Luo and W. Yu, "An introduction to convex optimization for communications and signal processing," IEEE Journal on Selected Areas in Commun., vol. 24, no. 8, pp. 1426-1438, Aug. 2006.

[26] S. Boyd and L. Vandenberghe, Convex Optimization. Cambridge, UK: Cambridge Univ. Press, 2004.

[27] M. Abramowitz and I. A. Stegun, Handbook of Mathematical Functions with formulas, Graphs and Mathematical Tables. New-York, Dover Press, Jun. 1974. 